\title{
Research Paper: The Validity and Reliability of Kinovea Software in Measuring Thoracic Kyphosis and Lumbar Lordosis
}

\author{
Ali Sharifnezhad ${ }^{1,2}$ (D), Gholam Reza Raissi ${ }^{3}$ (D), Bijan Forogh ${ }^{3}$ (D), Hosnieh Soleymanzadeh ${ }^{3}$, Shadan Mohammadpour ${ }^{4}$ (D), Mina Daliran ${ }^{5}$ (D), \\ Masumeh Bagherzadeh Cham $^{3,6^{*}}$ (1) \\ 1. Department of Sport Biomechanics and Technology, Sport Science Research Institute, Tehran, Iran. \\ 2. Neuromusculoskeletal Research Center, Iran University of Medical Sciences, Tehran, Iran. \\ 3. Neuromusculoskeletal Research Center, Department of Physical Medicine and Rehabilitation, School of Medicine, Firoozgar Hospital, Tehran, Iran \\ 4. Department of Health and Sports Medicine, Faculty of Physical Education and Sports Sciences, University of Tehran, Tehran, Iran. \\ 5. Department of Physical Education and Sport Sciences, Faculty of Humanities, North Tehran Branch, Islamic Azad University, Tehran, Iran. \\ 6. Department of Orthotics \& Prosthetics, Iran University of Medical sciences, Tehran, Iran.
}

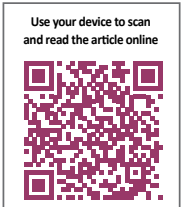

ditat On: Sharifnezhad A, Raissi GR, Forogh B, Soleymanzadeh H, Mohammadpour S, Daliran S, Bagherzadeh Cham M. The Validity and Reliability of Kinovea Software in Measuring Thoracic Kyphosis and Lumbar Lordosis. Iranian Rehabilitation Journal. 2021; 19(2):129-136. http://dx.doi.org/10.32598/irj.19.2.670.1

http://dx.doi.org/10.32598/irj.19.2.670.1

Article info:

Received: 03 Apr 2021

Accepted: 17 May 2021

Available Online: 01 June 2021

\section{Keywords:}

Validity, Reliability, Posturography, Kinovea software, Thoracic kyphosis, Lumbar lordosis

\section{A B STRACT}

Objectives: The present study evaluated the inter-rater and intra-rater validity and reliability of posturography by Kinovea software to measure the thoracic kyphosis and lumbar lordosis.

Methods: Eighteen subjects (10 females \& 8 males) referring for radiographic imaging were included in this cross-sectional study. For evaluating the validity, the thoracic kyphosis and lumbar lordosis were measured according to the Cobb method and Kinovea in standing position. The inter-rater and intra-rater reliability of Kinovea were tested by 3 evaluators and one expert evaluator, respectively.

Results: Pearson correlation coefficient data suggested that the validity of measuring the thoracic kyphosis depends on the evaluator's expertise. Besides, the correlation was not significant in measuring the lumbar lordosis angle $(\mathrm{P}>0.05)$. The inter-rater and intra-rater repeatability revealed that the correlation was significant in all angles by the intraclass correlation coefficient $(\mathrm{P}<0.001)$.

Discussion: Posturography by Kinovea, as a noninvasive method presents an excellent interrater and intra-rater repeatability for measuring thoracic kyphosis and lumbar lordosis. This reliable method is simple, efficient, and inexpensive.

\section{* Corresponding Author:}

Masumeh Bagherzadeh Cham, PhD.

Address: Neuromusculoskeletal Research Center, Department of Physical Medicine and Rehabilitation, School of Medicine, Firoozgar Hospital, Tehran, Iran. Tel: +98 (21) 82141612

E-mail: masumehbagherzadeh@gmail.com 


\section{Highlights}

- Kinovea is clinically suitable for evaluating thoracic kyphosis.

- The inter-rater and intra-rater reliability of Kinovea were measured to be high.

- Kinovea is inexpensive and safe to evaluate kyphosis and lordosis.

\section{Plain Language Summary}

Evaluating thoracic kyphosis and lumbar lordosis in all age groups is important to diagnose and treat musculoskeletal problems. The standard method of evaluation is radiographic imaging, which can be harmful on multiple radiographs. Using a simple camera and free Kinovea software for such evaluations is simple and low-risk. In this method, side photos were taken and the angle of thoracic kyphosis and lumbar lordosis were evaluated by Kinovea software by 3 testers with different skill levels. The present study results suggested that this method is suitable for evaluating thoracic kyphosis. Besides, the evaluation results of all 3 testers were the same and reliable.

\section{Introduction}

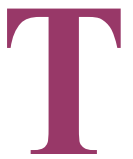

he spinal structure is usually evaluated to help guide the diagnosis and treatment of musculoskeletal problems [1] Thoracic kyphosis and lumbar lordosis are commonly evaluated when examining the spinal condition. Changes in the normal angle of thoracic kyphosis and lumbar lordosis are the most frequent spinal abnormalities [2]. Excessive kyphosis has been associated with musculoskeletal complaints, such as shoulder [3], cervical [4], and back pain [5], an increased risk of fracture [6], and impaired respiratory function [7]. Several studies signified a decreased lumbar lordosis angle as a leading cause of low back pain [8,9], increased risk of falls [10], and reduced Quality of Life (QoL) [11].

The accurate, early, and timely identification of these abnormalities can prevent, stop progression, and correct musculoskeletal disorders. The assessment and measurement of spinal curvatures are significant factors in corrective exercises and physiotherapy treatments. Moreover, the extent of curvature or progress usually determines the appropriate treatment method [12]. Measuring the angles of thoracic and lumbar curvature is possible using various invasive and non-invasive methods [13, 14]. Some invasive methods include radiographic images, Computerized Tomography (CT) scans, and Magnetic Resonance Imaging (MRI) [14]. The gold standard for evaluating the spinal curvature is using radiographs and the Cobb method [13,14]. Although this method is more reliable for assessing the spinal curvature, it is not especially suitable (due to its high cost, and radiation exposure) in pregnant women [15]. Studies suggested that this method increases prostate cancer risk in men [16] and breast cancer in women [17].

There exist several non-invasive methods for measuring the angle of thoracic kyphosis and lumbar lordosis. The validity of the flexible ruler has been reported; however, the degree of its repeatability remains controversial among studies; or a spinal mouse that requires a fixed device. Another non-invasive approach is applying two digital inclinometers. Besides, the reproducibility of this device varied in different studies [13, 14, 18]. Therefore, a valid, reliable, rapid, and non-invasive method for assessing kyphosis and lumbar lordosis is useful in clinical and research practices.

Kinovea is a free $2 \mathrm{D}$ motion analysis software under the GPL v2 license, developed in 2009 by the non-profit collaboration of several researchers, athletes, coaches, and programmers. Kinovea is available for download on the web $[19,20]$. It is portable, inexpensive, and feasible. Kinovea has been used along with $2 \mathrm{D}$ imaging by the digital camera for sports and clinical evaluations. The reliability and validity of this system were evaluated for measuring the range of motion in different joints of the body, including the shoulder [21], wrist [22], and cervical region [23]. Puig-Divi et al. examined the validity and reliability of this system for simultaneously evaluating different body angles [20]. However, this system's validity and reliability remain unevaluated for measuring the thoracic kyphosis and lumbar lordosis. Consequently, the current study aimed to evaluate the construct validity and inter-rater and intra-rater test-retest reliability of posturography by Kinovea to measure the thoracic kyphosis and lumbar lordosis. 


\section{Methods}

In this cross-sectional study, individuals were selected by a simple non-probability sampling method. The study subjects were selected from patients presented to the physiotherapy, physical medicine and rehabilitation, and orthopedics specialists at Firozgar Hospital, Tehran, Iran. The study subjects were referred to radiology centers for obtaining a lateral-view spine radiograph.

Including participants had no age limit. Only patients who had balance disorders when standing or were unable to stand without assistance; those with a history of a spinal tumor, surgery, radiotherapy, trauma, spinal fracture or skeletal disease, scoliosis deviation, leg length discrepancy, and psychiatric disorders were excluded from this study. All steps of the procedure were explained to the research participants or their guardians, and an informed consent form was obtained from them for digital camera photography. The Ethics Committee of Iran University of Medical Sciences approved this study (Code: IR.IUMS.REC 1396.32599).

In the present study, $\alpha=0.05$ and $\beta=0.1$ were considered and 3 testers performed the necessary assessments. Based on the previous studies, the minimum and the highest acceptable levels of reliability were determined as $\rho 0=70$ and $\rho 1=0.90$, respectively. These values were placed in a table provided by Walter et al. [24] to calculate the required sample size in reliability studies. As a result, the sample size of 18 participants was computed for this research.

Measuring the Cobb angle requires a full-body radiograph on the sagittal plane. The study subject was requested to stand barefoot with a comfortable and natural position on the cardboard, where the foot placement area was marked. They were also requested to keep their shoulders and elbows at $90^{\circ}$ of flexion to avoid the spine's upper limb shadow [25]. The explored patient's code was then provided to a physical medicine and rehabilitation specialist; accordingly, after measuring the kyphosis and lordosis angle using a camera and Kinovea, the researcher was provided with the relevant results.

The Cobb method was employed to determine the angles and degrees of the kyphosis and lordosis in radiographs. Kyphosis was determined using a Cobb angle between the superior vertebral endplate of $\mathrm{T} 4$ and the inferior vertebral endplate of T12 [26]. Furthermore, lumbar lordosis was analyzed using lines drawn along the superior vertebral endplate of L1 and the inferior vertebral endplate of L5 [27]. Then, lines perpendicular to each of these lines were drawn, and the acute angle of their intersection was calculated, which indicated the degrees of kyphosis and lordosis (Figure 1) [9].

To measure the angle of kyphosis and lordosis by Kinovea, a complete image of the subject in the lateral view is required. For this purpose, the study subjects were instructed to take off their clothes, and a specialist marked bilateral earlobes.

The examined individuals were requested to stand sideways and barefoot on the back of the calibration plane with shoulders and elbows at $90^{\circ}$-flexion (similar to radiographs). Their legs were leveled and sided, and their weight evenly distributed on two legs, and they looked at a fixed point on the opposite wall. Each study subject had to remain in this position for 2 minutes to achieve their normal position; the shooting was repeated 3 times during this time.

The shooting was conducted using a Sony digital camera placed 1.5 meters away from the calibration plane and at a one-meter height from the ground on a tripod [22]. The camera was adjusted using the level placed on the tripod. The camera lens had to be placed perpendicular to the calibration plane. This is because the measurement was performed in $2 \mathrm{D}$.

The angles of the kyphosis and lordosis were calculated using Kinovea. Initially, a line was drawn parallel to the calibration plane from the earmark, i.e., considered the plumb line. To calculate the kyphosis angle, the farthest point from the plumb line was determined. This point is considered the apex of the kyphosis angle. Then, two lines at the top and bottom were drawn parallel to the curvature of the back. These lines intersected at the apex of each other. The angle of intersection of the lines was measured by Kinovea and decreased from $180^{\circ}$. The calculated value was reported as the kyphosis angle (Figure 2).

To calculate the lordosis angle, the nearest point from the plumb line was determined. Then, two lines at the top and bottom were drawn parallel to the curvature of the lumbar. The angle of the intersection of the lines was measured by Kinovea and decreases from $180^{\circ}$. The calculated angle was reported as the lordosis angle (Figure 2).

First, one of the 3 images taken in the Kinovea evaluation process was randomly selected and anonymously provided to the evaluators. The 3 evaluators separately measured the angles of kyphosis and lordosis using Kinovea. The first evaluator was a specialist with $>20$ years of experience, the second evaluator was a special- 
A

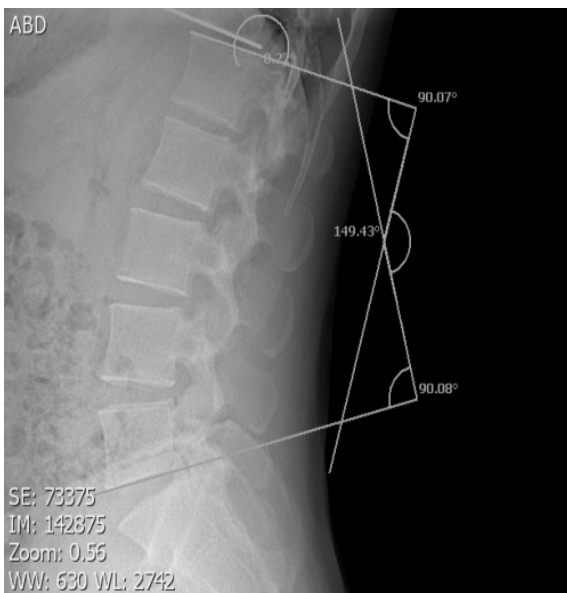

B

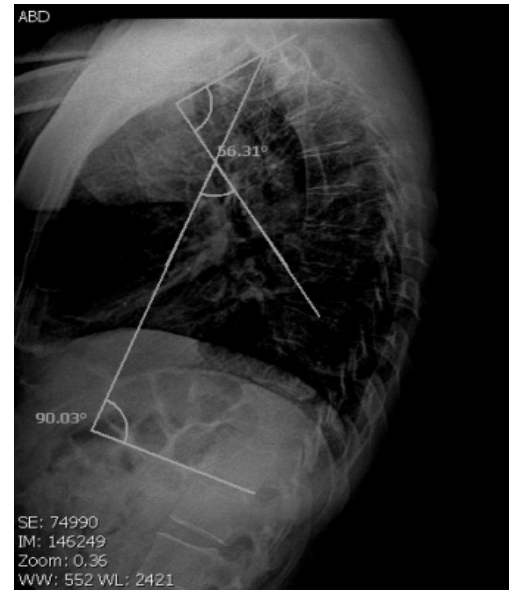

Iranian Rehabilitation Journal

Figure 1. Measuring thoracic kyphosis (A) and lumbar lordosis (B) by the Cobb method

subjects are listed in Table 1. The Kolmogorov-Smirnov test data signified that the data had a normal distribution. The obtained Pearson correlation coefficient to determine the validity of Kinovea in measuring the thoracic kyphosis angles indicated that the validity of measuring the kyphosis depended on the evaluator's expertise. Besides, the correlation was not significant in measuring the lumbar lordosis angle $(\mathrm{P}>0.05)$ (Table 2).

The inter-rater and intra-rater repeatability calculated by Kinovea concerning kyphosis and lordosis angles revealed that the correlation was significant in all angles by the ICC $(\mathrm{P}<0.001)$. The results of inter-rater and intrarater repeatability are presented in Table 3 .

\section{Discussion}

The present study results suggested that using posturography by Kinovea for measuring the angle of kyphosis and lordosis presented a high inter and intra-rater repeatability; however, its validity depends on the examiners' skills, compared with the Cobb method. Exploring the validity and reliability of measuring methods plays an essential role in establishing a solid foundation for research results or clinical evaluation findings. Currently, the gold standard for measuring kyphosis and lordosis is the X-ray method. However, problems, such as cost, time-consuming, potential risks, and difficulty in the imaging's repetition have made it an unsuitable approach $[13,14]$.

A review by Barrett et al. classified 15 non-invasive methods for assessing thoracic kyphosis, ranging from simple to complex computer methods. In most of these methods, the reliability was high or very high; however, the validity of these techniques remains unexamined. Moreover, in the limited studied cases, this range has ranged from low to very high. It also does not seem that 
Table 1. Demographic information of the study subjects

\begin{tabular}{cccc}
\hline Variables & Mean \pm SD & Min. & Max. \\
\hline Age $(\mathrm{y})$ & $42.64 \pm 21.67$ & 9 & 73 \\
\hline Height $(\mathrm{cm})$ & $164.0 \pm 11.8$ & 138 & 180 \\
\hline Weight $(\mathrm{kg})$ & $64.06 \pm 14.53$ & 30 & 85 \\
BMl $\left(\mathrm{kg} / \mathrm{m}^{2}\right)$ & $23.48 \pm 3.62$ & 15.75 & 29.38 \\
\hline
\end{tabular}

Iranian Rehabilitation Journa

complicated computer methods are more valid and reliable than more straightforward approaches [18].

Kinovea is a free 2D motion analysis software. This inexpensive technology has been used in sports science as well as clinical and research studies. Studies using Kinovea to measure the joints' range of motion revealed that this system has a high inter-rater and intra-rater reliability. Besides, the testers' background and expertise present no effect on its reliability [20-23]. The present study results also indicated that the intra-group repeatability of Kinovea is high in measuring the kyphosis $(\mathrm{ICC}=0.90)$ and lordosis $(\mathrm{ICC}=0.91)$. The inter-group reliability of Kinovea in measuring the thoracic kyphosis $(\mathrm{ICC}=0.81)$ and lumbar lordosis $(\mathrm{ICC}=0.87)$ was also high.

A potential challenge in assessing reliability is the precise palpation of the spine. The accuracy of the palpation results throughout the spine has been reported to be low $[18,28]$. This could also be affected by the examiner's skills [28]. In this study, spinal palpation and specialized marking were unnecessary. This is because only the earlobes were marked to determine the plumb line, which requires no expertise.
Another factor affecting the measuring methods' reliability is the changes that may occur over time, such as fatigue due to repeated tests or exercise between two repeated sessions $[18,29]$. In this study, each subject was photographed only once. Besides, 3 evaluators examined the images, and the angle of kyphosis and lordosis were calculated. Therefore, environmental factors did not affect the repeatability of the data. However, it is better to repeat the imaging in different sessions to evaluate the reliability of Kinovea in measuring the angle of thoracic kyphosis and lumbar lordosis.

An advantage of Kinovea is that the examiner or measuring instrument does not contact the subjects; thus, it can reduce the odds of error. This is because some factors, like the pressure on the spine by a measuring instrument in tools, such as flexicurve and spinal mouse can affect the reliability [18, 29-31]. As a result, Kinovea seems to be more accurate than other contact methods; with one shot, the thoracic kyphosis and lumbar lordosis angles can be measured in this manner.

There are access restrictions and ethical issues regarding the use of X-rays to examine the validity of the

Table 2. Comparing correlation values between Cobb method and Kinovea software for all evaluators

\begin{tabular}{|c|c|c|c|c|}
\hline \multicolumn{2}{|c|}{ Variables } & Mean \pm SD & Pearson Correlation Coefficient & $\mathbf{P}$ \\
\hline \multirow{4}{*}{ Thoracic kyphosis } & First evaluator & $29.0 \pm 7.35$ & 0.48 & 0.049 \\
\hline & Second evaluator & $28.5 \pm 7.43$ & 0.59 & 0.013 \\
\hline & Third evaluator & $31.5 \pm 7.78$ & 0.317 & 0.21 \\
\hline & Cobb angle & $49.4 \pm 13.13$ & & \\
\hline \multirow{4}{*}{ Lumbar lordosis } & First evaluator & $34.6 \pm 8.8$ & 0.38 & 0.13 \\
\hline & Second evaluator & $33.8 \pm 8.7$ & 0.40 & 0.10 \\
\hline & Third evaluator & $36.1 \pm 8.25$ & 0.34 & 0.18 \\
\hline & Cobb angle & $40.6 \pm 11.52$ & & \\
\hline
\end{tabular}


Table 3. Reliability of the Kinovea software in measuring thoracic kyphosis and lumbar lordosis

\begin{tabular}{ccccc}
\hline & \multicolumn{2}{c}{ Intra-rater } & \multicolumn{3}{c}{ Inter-rater } \\
\cline { 2 - 5 } Variables & ICC & $\mathbf{P}$ & ICC & $\mathbf{P}$ \\
\hline Thoracic kyphosis & 0.90 & $<0.001$ & 0.81 & $<0.001$ \\
Lumbar lordosis & 0.91 & $<0.001$ & 0.87 & $<0.001$ \\
\hline
\end{tabular}

kyphosis and lordosis measurement method [32]. This study only included individuals referring for sagittal radiographs for clinical evaluation. The results indicated that the validity of Kinovea in measuring thoracic kyphosis depended on the expertise of the evaluator; only a specialist evaluator $(\mathrm{r}=0.48, \mathrm{P}=0.049)$ and a trained expert with 5 years of experience $(\mathrm{r}=0.59, \mathrm{P}=0.013)$ provided an acceptable validity, compared to the Cobb method. Furthermore, Kinovea has no acceptable validity in measuring the lumbar lordosis $(\mathrm{P}>0.05)$.

In measuring the angle of thoracic kyphosis and lumbar lordosis, vertebrae bodies are examined by the standard Cobb method; however, other non-invasive methods use the palpable vertebral spinous processes for the measurement $[18,30]$. Muscles and the adipose tissue located on the vertebrae make it difficult to palpate the vertebrae and affect the measurement method's accuracy [30]. The Kinovea required no vertebrae palpation; however, the

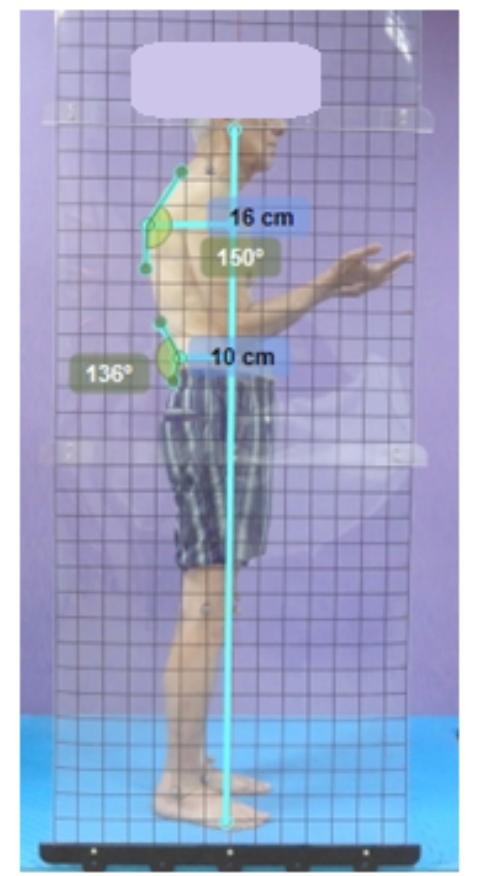

Iranian Rehabilitation Journa

Figure 2. Measuring thoracic kyphosis and lumbar lordosis by Kinovea software adipose tissue or muscle weakness was an influencing factor in measuring thoracic kyphosis and lumbar lordosis.

Most studies evaluated healthy individuals aged between 20 and 65 years. A healthy population in this age group does not necessarily represent a clinical population [33]; therefore, the results cannot be generalized to the clinical populations. Greendale et al. [34] and Yanagawa et al. [35] used Flexicurve in postmenopausal osteoporosis to assess its validity and reliability, thereby increasing the Flexicurve index's credibility. In the present study, individuals with different problems participated without age restrictions, which can affect the validity of the method.

The literature suggests that the accuracy of the lumbar lordosis angle measuring equipment is less than that of the thoracic kyphosis. This is because of the difference in the high mobility of the target areas $[13,25]$. In the chest, flexion/extension at the sagittal plane is limited by the joints; however, the thoracic vertebrae benefit from a more extended flexion. Besides, as the measurement is not performed simultaneously, the lumbar lordosis is subject to much more changes, compared to kyphosis for the subject's movement [25]. However, in the present study, images were obtained while standing and simultaneously from the thoracic kyphosis and lumbar lordosis. Nevertheless, the collected results demonstrated that Kinovea presented no acceptable validity in measuring lumbar lordosis.

This study's main limitations were the small and heterogeneous sample size and the wide age range. This is because obtaining unreasonable X-rays is morally incorrect and may be harmful to the subjects. Therefore, in this study, only patients undergoing spine lateral view imaging for clinical reasons were included.

It is recommended to check the repeatability in repeated attempts. Additionally, evaluating the validity and reliability using larger sample size and selecting subjects with different age groups and problems are recommended. The validity and reproducibility of Kinovea can also 
be evaluated for measuring other spinal abnormalities, such as scoliosis or lumbar lordosis.

\section{Conclusion}

Kinovea software has excellent inter-rater and intrarater repeatability $(\mathrm{ICC}<0.80)$ for measuring kyphosis and lordosis. The advantage of using this reliable method is that it is simple, efficient, and inexpensive. This method's use is not affected by spinal palpation, applying force on the spine, or marking skills. This method can be employed in repeated follow-ups and the evaluation of the clinical treatment progress due to these positive features. This is the first study that has used this software to examine the thoracic kyphosis and lumbar lordosis; thus, further studies with a larger sample size are recommended to evaluate the validity and reliability of this method.

\section{Ethical Considerations}

\section{Compliance with ethical guidelines}

The Ethics Committee of Iran University of Medical Sciences approved this study (Code: IR.IUMS.REC 1396.32599).

\section{Funding}

This research was supported by Iran University of Medical Sciences (grant No.: 32599).

\section{Authors' contributions}

Conceptualization: Ali Sharifnezhad, Bijan Forogh, Masumeh Bagherzadeh Cham; Methodology: Ali Sharifnezhad, Gholamreza Raissi, Bijan Forogh, Masumeh Bagherzadeh Cham; Software: Ali Sharifnezhad, Gholamreza Raissi, Hosniyeh Soleymanzadeh, Shadan Mohammadpour, Mina Daliran; Validation: Ali Sharifnezhad, Gholamreza Raissi, Shadan Mohammadpour, Mina Daliran; Formal Analysis: Masumeh Bagherzadeh Cham, Hosniyeh Soleymanzadeh; Investigation Ali Sharifnezhad, Gholamreza Raissi, Bijan Forogh, Hosniyeh Soleymanzadeh, Masumeh Bagherzadeh Cham; Resources: Masumeh Bagherzadeh Cham, Hosniyeh Soleymanzadeh; Data Curation: Hosniyeh Soleymanzadeh; Writing - Original Draft Preparation: Ali Sharifnezhad, Bijan Forogh, Masumeh Bagherzadeh Cham; Writing - Review \& Editing: Ali Sharifnezhad, Gholamreza Raissi, Bijan Forogh, Masumeh Bagherzadeh Cham; Supervision: Ali Sharifnezhad, Gholamreza Raissi, Bijan Forogh, Masumeh Bagherzadeh Cham;
Project Administration: Ali Sharifnezhad, Gholamreza Raissi, Bijan Forogh, Masumeh Bagherzadeh Cham.

\section{Conflict of interest}

The authors declared no conflicts of interest.

\section{Acknowledgments}

This research was supported with Grant No. 32599 by Iran University of Medical Sciences.

\section{References}

[1] Kuo YL, Tully EA, Galea MP. Sagittal spinal posture after Pilates-based exercise in healthy older adults. Spine. 2009; 34(10):1046-51. [DOI:10.1097/BRS.0b013e31819c11f8] [PMID]

[2] Britnell SJ, Cole JV, Isherwood L, Stan MM, Britnell N, Burgi $\mathrm{S}$, et al. Postural health in women: The role of physiotherapy. Journal of Obstetrics and Gynaecology Canada. 2005; 27(5):493-500. [DOI:10.1016/S1701-2163(16)30535-7]

[3] Gray J, Grimsby O. Interrelationship of the spine, rib cage, and shoulder. In: Donatelli RA, editor. Physical Therapy of the Shoulder. Amsterdam: Elsevier Health Sciences; 2004. pp. 133-85. [DOI:10.1016/B978-044306614-6.50007-7]

[4] Joshi S, Balthillaya G, Neelapala YVR. Thoracic posture and mobility in mechanical neck pain population: A review of the literature. Asian Spine Journal. 2019; 13(5):849-60. [DOI:10.31616/asj.2018.0302] [PMID] [PMCID]

[5] Ensrud KE, Black DM, Harris F, Ettinger B, Cummings SR Correlates of kyphosis in older women. The Fracture Intervention Trial Research Group. Journal of the American Geriatrics Society. 1997; 45(6):682-7. [DOI:10.1111/j.1532-5415.1997. tb01470.x] [PMID]

[6] Huang MH, Barrett-Connor E, Greendale GA, Kado DM Hyperkyphotic posture and risk of future osteoporotic fractures: The Rancho Bernardo study. Journal of Bone and Mineral Research:The Official Journal of the American Society for Bone and Mineral Research. 2006; 21(3):419-23. [DOI:10.1359/ JBMR.051201] [PMID] [PMCID]

[7] Di Bari M, Chiarlone M, Matteuzzi D, Zacchei S, Pozzi C, Bellia V, et al. Thoracic kyphosis and ventilatory dysfunction in unselected older persons: An epidemiological study in Dicomano, Italy. Journal of the American Geriatrics Society. 2004; 52(6):909-15. [DOI:10.1111/j.1532-5415.2004.52257.x][PMID]

[8] Smith A, O'Sullivan P, Straker L. Classification of sagittal thoraco-lumbo-pelvic alignment of the adolescent spine in standing and its relationship to low back pain. Spine. 2008; 33(19):2101-7. [DOI:10.1097/BRS.0b013e31817ec3b0][PMID]

[9] Sorensen CJ, Norton BJ, Callaghan JP, Hwang C-T, Van Dillen LR. Is lumbar lordosis related to low back pain development during prolonged standing? Manual Therapy. 2015; 20(4):553-7. [DOI:10.1016/j.math.2015.01.001] [PMID][PMCID] 
[10] Ishikawa Y, Miyakoshi N, Kasukawa Y, Hongo M, Shimada Y. Spinal sagittal contour affecting falls: Cut-off value of the lumbar spine for falls. Gait \& Posture. 2013; 38(2):260-3. [DOI:10.1016/j.gaitpost.2012.11.024] [PMID]

[11] Imagama S, Hasegawa Y, Matsuyama Y, Sakai Y, Ito Z Hamajima $N$, et al. Influence of sagittal balance and physical ability associated with exercise on quality of life in middle-aged and elderly people. Archives of Osteoporosis. 2011; 6(1-2):13-20 [DOI:10.1007/s11657-011-0052-1] [PMID] [PMCID]

[12] Chaise FO, Candotti CT, Torre ML, Furlanetto TS, Pelinson PP, Loss JF. Validation, repeatability and reproducibility of a noninvasive instrument for measuring thoracic and lumbar curvature of the spine in the sagittal plane. Revista Brasileira de Fisioterapia (Sao Paulo, Brazil). 2011; 15(6):511-7. [DOI:10.1590/S1413-35552011005000031] [PMID]

[13] Sedrez JA, Candotti CT, Furlanetto TS, Loss JF. Non-invasive postural assessment of the spine in the sagittal plane: A systematic review. Journal Motricidade. 2016; 12(2):140-54 [DOI:10.6063/motricidade.6470]

[14] Cohen L, Kobayashi S, Simic M, Dennis S, Refshauge K, Pappas E. Non-radiographic methods of measuring global sagittal balance: A systematic review. Scoliosis Spinal Disord. 2017 12:30. [DOI:10.1186/s13013-017-0135-x] [PMID] [PMCID]

[15] Legaye J. Follow-up of the sagittal spine by optical technique. Annals of Physical and Rehabilitation Medicine. 2012 55(2):76-92. [DOI:10.1016/j.rehab.2011.12.002] [PMID]

[16] Myles P, Evans S, Lophatananon A, Dimitropoulou P, Easton D, Key T, et al. Diagnostic radiation procedures and risk of prostate cancer. British Journal of Cancer. 2008; 98(11):1852-6. [DOI:10.1038/sj.bjc.6604370] [PMID] [PMCID]

[17] John EM, Phipps AI, Knight JA, Milne RL, Dite GS, Hopper JL, et al. Medical radiation exposure and breast cancer risk: Findings from the Breast Cancer Family Registry. International Journal of Cancer. 2007; 121(2):386-94. [DOI:10.1002/ijc.22668] [PMID]

[18] Barrett E, McCreesh K, Lewis J. Reliability and validity of non-radiographic methods of thoracic kyphosis measurement: A systematic review. Manual Therapy. 2014; 19(1):10-7. [DOI:10.1016/j.math.2013.09.003] [PMID]

[19] Kinovea. Features [Internet]. 2021 [Updated 20 June 2021]. Available from: http:/ / www.kinovea.org

[20] Puig-Diví A, Escalona-Marfil C, Padullés-Riu JM, Busquets A, Padullés-Chando X, Marcos-Ruiz D. Validity and reliability of the Kinovea program in obtaining angles and distances using coordinates in 4 perspectives. PLoS ONE. 2019; 14(6):e0216448. [DOI:10.1371/journal.pone.0216448] [PMID][PMCID]

[21] Abd Elrahim R, Embaby E, Ali M, Kamel R. Inter-rater and intra-rater reliability of Kinovea software for measurement of shoulder range of motion. Bulletin of Faculty of Physical Therapy. 2016; 21(2):80-7. [DOI:10.4103/1110-6611.196778]

[22] Abd El-Raheem RM, Kamel RM, Ali MF. Reliability of using kinovea program in measuring dominant wrist joint range of motion. Trends in Applied Sciences Research. 2015; 10(4):224-30. [DOI:10.3923/tasr.2015.224.230]

[23] Elwardany SH, Eleiny KE, Arabia S. Reliability of kinovea computer program in measuring cervical range of motion in sagittal plane. Open Access Library Journal. 2015; 2(09):1-10. [DOI:10.4236/oalib.1101916
[24] Walter SD, Eliasziw M, Donner A. Sample size and optimal designs for reliability studies. Statistics in Medicine. 1998; 17(1):101-10. [DOI:10.1002/(SICI)1097-0258(19980115)17:13.0.CO;2-E]

[25] Leroux MA, Zabjek K, Simard G, Coillard C, Rivard CH. Estimated kyphosis and lordosis changes at follow-up in patients with idiopathic scoliosis. Journal of Pediatric Orthopedics. 2002; 22(1):73-9. [PMID]

[26] Lorbergs AL, Murabito JM, Jarraya M, Guermazi A, Allaire BT, Yang L, et al. Thoracic kyphosis and physical function: The framingham study. Journal of the American Geriatrics Society. 2017; 65(10):2257-64. [DOI:10.1111/jgs.15038] [PMID][PMCID]

[27] Russell BS, Muhlenkamp-Wermert KA, Hoiriis KT. Measurement of Lumbar lordosis: A comparison of 2 alternatives to the cobb angle. Journal of Manipulative and Physiological Therapeutics. 2020; 43(8):760-7. [DOI:10.1016/j. jmpt.2020.05.009] [PMID]

[28] Rosner B. The intraclass correlation coeffiient. In: Rosner B editor. Fundamental of Biostatistics. Boston, Massachusetts: Cengage Learning. 2016; p. 569. https:/ / www.google.com/books/ edition/Fundamentals_of_Biostatistics/1o1izgEACAAJ?hl=en

[29] Billis EV, Foster NE, Wright CC. Reproducibility and repeatability: Errors of three groups of physiotherapists in locating spinal levels by palpation. Manual Therapy. 2003; 8(4):223-32. [DOI:10.1016/S1356-689X(03)00017-1]

[30] Kellis E, Adamou G, Tzilios G, Emmanouilidou M. Reliability of spinal range of motion in healthy boys using a skin-surface device. Journal of Manipulative and Physiological Therapeutics. 2008; 31(8):570-6. [DOI:10.1016/j.jmpt.2008.09.001] [PMID]

[31] Mannion AF, Knecht K, Balaban G, Dvorak J, Grob D. A new skin-surface device for measuring the curvature and global and segmental ranges of motion of the spine: Reliability of measurements and comparison with data reviewed from the literature. European Spine Journal. 2004; 13(2):122-36. [DOI:10.1007/s00586-003-0618-8] [PMID] [PMCID]

[32] Hinman M. Interrater reliability of flexicurve postural measures among novice users. Journal of Back and Musculoskeletal Rehabilitation. 2004; 17(1):33-6. [DOI:10.3233/BMR-2004-17107]

[33] Whiting P, Rutjes AW, Reitsma JB, Bossuyt PM, Kleijnen J. The development of QUADAS: A tool for the quality assessment of studies of diagnostic accuracy included in systematic reviews. BMC Medical Research Methodology. 2003; 3:25. [DOI:10.1186/1471-2288-3-25] [PMID] [PMCID]

[34] Greendale GA, Nili NS, Huang MH, Seeger L, Karlamangla AS. The reliability and validity of three non-radiological measures of thoracic kyphosis and their relations to the standing radiological Cobb angle. Osteoporosis International. 2011; 22(6):1897-905. [DOI:10.1007/s00198-010-1422-z] [PMID] [PMCID]

[35] Yanagawa TL, Maitland ME, Burgess K, Young L, Hanley D. Assessment of thoracic kyphosis using the flexicurve for individuals with osteoporosis. Hong Kong Physiotherapy Journal. 2000; 18(2):53-7. [DOI:10.1016/S1013-7025(00)18004-2] 